\title{
ROS1 Asp2213Asn polymorphism is not associated with coronary artery disease in a Greek case-control study
}

\author{
Eirini V. Theodoraki ${ }^{1, *}$, Tiit Nikopensius ${ }^{2,3}$, \\ Julia Suhorutšenko ${ }^{2}$, Vassileios Papamikos ${ }^{1}$, \\ Genovefa D. Kolovou“ ${ }^{4}$, Vassileios Peppes ${ }^{5}$, \\ Demosthenes Panagiotakos ${ }^{1}$, Sasa Limberi ${ }^{6}$, \\ Nikolaos Zakopoulos ${ }^{5}$, Andres Metspalu Z $^{2,3,7}$ \\ and George V. Dedoussis ${ }^{1}$ \\ ${ }^{1}$ Department of Dietetics and Nutritional Science, \\ Harokopio University of Athens, Athens, Greece \\ ${ }^{2}$ Department of Biotechnology, University of Tartu, \\ Tartu, Estonia \\ ${ }^{3}$ Estonian Biocentre, Tartu, Estonia \\ ${ }^{4}$ Onassis Cardiac Surgery, 1st Cardiology Clinic, \\ Athens, Greece \\ ${ }^{5}$ Department of Clinical Therapeutics, University of \\ Athens Medical School "Alexandra Hospital", \\ Athens, Greece \\ ${ }^{6}$ Cardiology Clinic, Sotiria Hospital, Athens, Greece \\ ${ }^{7}$ Estonian Genome Project, University of Tartu, \\ Tartu, Estonia
}

\begin{abstract}
Background: Rs619203 (Cys2229Ser) and rs529038 (Asp2213Asn) polymorphisms in the ROS1 gene have been studied in relation to myocardial infarction (MI) yielding inconsistent results. We investigated the role of ROS1 rs529038 polymorphism in coronary artery disease (CAD) in Greeks using a case-control study. Methods: Genotyping for rs529038 polymorphism was performed using a multiplex PCR technique in patients with CAD $(n=294)$ and controls $(n=311)$. Logistic regression analysis was used to calculate crude and adjusted odds ratios (ORs).

Results: Logistic regression analysis did not show any statistically significant effect of ROS1 polymorphism in the occurrence of CAD ( $A G$ vs. AA, OR: 1.08, $p=0.635$; $G G$ vs. $A A$, OR: 0.62, $p=0.220$ ). Adjustment for confounding factors gave similar results, irrespective of the type of disease (i.e., stable coronary artery disease vs. acute coronary syndrome).

Conclusions: Our findings do not support the hypothesis that ROS1 rs529038 polymorphism is an important contributing factor in the etiology of CAD in the Greek population.

Clin Chem Lab Med 2009;47:1471-3.

\footnotetext{
*Corresponding author: Eirini Theodoraki, 70, El. Venizelou Street, 17671, Athens, Greece

Phone: + 302109549137, Fax: + 302109577050,

E-mail: itheodoraki@gmail.com

Received January 7, 2009; accepted March 24, 2009;

previously published online October 28, 2009
}

Keywords: acute coronary syndrome; atherosclerosis; coronary artery disease; growth factor receptor; nonsynonymous polymorphism.

\section{Introduction}

V-ros UR2 sarcoma virus oncogene homolog 1 (ROS1) gene is located in $6 \mathrm{q} 22$ and encodes for a proto-oncogene. This product is also a type I integral membrane protein with tyrosine kinase activity that may function as a growth or differentiation factor receptor and is expressed in most tissues $(1,2)$.

The ROS 1 gene was initially implicated in the etiology of myocardial infarction (MI) in a genome-wide association study. This study demonstrated that nonsynonymous rs529038 and rs619203 polymorphisms, which are almost in complete linkage disequilibrium, were important factors for risk of disease (3). To our knowledge, there are only two additional studies that attempted to replicate these findings. These studies showed inconsistent results in Caucasians of nonEuropean origin $(4,5)$.

These particular polymorphisms also have been shown to increase the risk for restenosis following coronary stenting, as well as for hypertension and atherothrombotic cerebral infarction in Japanese (6-8).

The aim of the present study was to investigate whether ROS1 rs529038 polymorphism is a significant contributor to coronary atherosclerosis in a Greek population. We employed a case-control study design using 294 patients with coronary artery disease (CAD) and 311 control subjects.

\section{Materials and methods}

\section{Subjects}

Study participants were unrelated individuals, exclusively of Greek origin, and were selected consecutively from hospitals in the Athens area. Subjects presenting with either acute coronary syndrome (ACS) or stable CAD were recruited as study cases. CAD was defined as $>50 \%$ stenosis in at least one of the three main coronary vessels, as assessed by coronary angiography. ACS was defined as: (1) acute MI, or (2) unstable angina corresponding to class III of the Braunwald classification (9). Controls were subjects with negative findings following coronary angiography, or negative stress test, or subjects without symptoms of disease who were admitted to the same hospitals as cases and were free of cardiovascular disease, cancer, or inflammatory diseases. The Institutional Ethics and Research Committee approved the research protocol and all participants gave their informed consent before enrollment in the study. 


\section{Risk factor definition}

Subjects defined as hypercholesterolemic had either total cholesterol levels $>200 \mathrm{mg} / \mathrm{dL}(>5.128 \mathrm{mmol} / \mathrm{L})$, or were under treatment for hyperlipidemia. Subjects whose blood pressure was $\geq 140 / 90 \mathrm{~mm} \mathrm{Hg}$, or who were taking antihypertensive medication were classified as having hypertension. Individuals with a fasting blood glucose $>125 \mathrm{mg} / \mathrm{dL}$ ( $>5.277 \mathrm{mmol} / \mathrm{L}$ ), or those requiring a special diet or treatment where classified as diabetics. Positive family history was defined as the presence of premature $\mathrm{MI}$ among firstdegree relatives $(<55$ years for male relatives and $<65$ for female relatives). Smokers and non-smokers were grouped together.

\section{Genotyping}

Genomic DNA was extracted from whole blood using the salting-out method (10). Since the above single nucleotide polymorphisms (SNPs) are in almost complete linkage disequilibrium, we genotyped for rs529038 polymorphism only. Rs529038 was detected among other SNPs, using an arrayed primer extension-based genotyping method (APEX-2). This method allows multiplex DNA amplification and detection of SNPs on microarrays using four-color single-base primer extensions (11). The specific primers for rs529038 polymorphism were:

\section{Left: GCTATTTAAGAAAAAATTTCTGAATAACTGAAGTTGGT} Right: CGACCAAAGACCTACTTTTCATAGAATTCAG

\section{Statistical analysis}

The $\chi^{2}$-test was used to compare the observed numbers of each genotype with the values expected for a population in Hardy-Weinberg equilibrium. Continuous variables are shown as mean $\pm S D$, while categorical variables are presented as percentages. Differences in genotype distributions between patients and controls were evaluated using the $\chi^{2}$ test. Odds ratios (ORs) were calculated using logistic regression. Multivariate logistic regression analysis was used to calculate the adjusted OR. Variables used included age, sex, and the prevalence of smoking, diabetes, hypertension, hypercholesterolemia and family history of MI. Two-sided tests were performed and a $\mathrm{p}$-value $\leq 0.05$ was used for statistical significance. Statistical analyses were performed using SPSS 13.0 (SPSS Inc., Chicago, IL, USA).

Power analysis was performed using QUANTO 1.2 software under the assumption of the additive model of inheritance.

\section{Results}

Demographics of participants are presented in Table 1. Mean age and the percentage of male subjects were slightly higher in patients compared to controls. As expected, patients exhibited a higher prevalence of diabetes, hypercholesterolemia, hypertension, family history of $\mathrm{Ml}$ and smoking compared to controls. Body mass index (BMI) did not differ significantly between the two study groups.

The distribution of ROS1 rs529038 polymorphism in controls was comparable with the Hardy Weinberg equilibrium $(p=0.93)$. Table 2 shows the distribution of genotypes between patients and controls and results of the logistic regression analysis that assessed the relationship between ROS1 rs529038 polymorphism and the likelihood of having CAD (presence or absence of ACS). The latter analysis was performed both before and after controlling for the effect of several potential confounders such as age, sex, and prevalence of smoking, diabetes, hypertension, hypercholesterolemia and family history of MI. There was no statistically significant effect of rs529038 polymorphism on the occurrence of CAD. In addition, the lack of association was not influenced by the type of disease (stable CAD vs. ACS) ( $p$ for

Table 1 Characteristics of the study subjects.

\begin{tabular}{|c|c|c|c|}
\hline Subject characteristics & $\begin{array}{l}\text { Cases } \\
(n=294)\end{array}$ & $\begin{array}{l}\text { Controls } \\
(\mathrm{n}=311)\end{array}$ & $\mathrm{p}$-Value \\
\hline Age, years & $63.8 \pm 10.8$ & $60.7 \pm 14.9$ & 0.002 \\
\hline Male sex, \% & 81.7 & 70.2 & 0.001 \\
\hline BMI, kg/m² & $28.0 \pm 3.9$ & $28.2 \pm 4.6$ & 0.580 \\
\hline Hypercholesterolemia, \% & 78.1 & 59.0 & $<0.001$ \\
\hline Diabetes, \% & 30.2 & 15.1 & $<0.001$ \\
\hline Hypertension, \% & 73.5 & 58.8 & $<0.001$ \\
\hline Family history of MI, \% & 27.9 & 16.7 & 0.002 \\
\hline Current or former smoking, \% & 74.8 & 61.0 & $<0.001$ \\
\hline
\end{tabular}

$\mathrm{BMI}$, body mass index; MI, myocardial infarction.

Table 2 Logistic regression analysis for the effect of ROS1 rs529038 polymorphism in coronary artery disease occurrence (presence or absence of acute coronary syndrome).

\begin{tabular}{llllll}
\hline SNP & Genotype & $\begin{array}{l}\text { Cases } \\
(\mathrm{n}=294)\end{array}$ & $\begin{array}{l}\text { Controls } \\
(\mathrm{n}=311)\end{array}$ & $\begin{array}{l}\text { Crude OR; } \\
95 \% \mathrm{Cl} ; \mathrm{p} \text {-value }\end{array}$ & \begin{tabular}{l}
$\begin{array}{l}\text { Adjusted OR; } \\
95 \% \mathrm{Cl} ; \mathrm{p} \text {-value }\end{array}$ \\
\hline ROS1 rs529038
\end{tabular} \\
& AA & $57.1 \%$ & $57.6 \%$ & Referent & Referent \\
& AG & $39.1 \%$ & $36.3 \%$ & $1.08 ; 0.78-1.52 ; 0.635$ & $0.96 ; 0.65-1.41 ; 0.818$ \\
& GG & $3.7 \%$ & $6.1 \%$ & $0.61 ; 0.28-1.34 ; 0.220$ & $0.51 ; 0.20-1.31 ; 0.160$ \\
\hline
\end{tabular}

Age, sex, prevalence of smoking, diabetes, hypertension, hypercholesterolemia and family history for myocardial infarction were used as covariates. Unadjusted and adjusted odds ratios (ORs), 95\% confidence intervals (Cl) and p-values were calculated using AA as referent genotype. 
interaction between type of disease and genotype $=0.9$ ).

\section{Discussion}

The aim of the present study was to investigate the role of ROS1 rs529038 polymorphism in the development of CAD in Greek adults. We did not observe any statistically significant effect of the polymorphism on the occurrence of disease, irrespective of the presence of ACS.

The minor allele frequency in the control group is 0.242 , which is in accordance with the frequencies reported in previous studies (0.21-0.27) (3-5).

Rs529038 and rs619203 polymorphisms, along with four more variants in other genes, were first reported to be associated with $\mathrm{Ml}$ in a genome-wide association study (3). Among these reported variants, only the association of ROS1 polymorphisms was replicated in the unadjusted analysis of a second study that utilized a prospective study design. However, the observed association disappeared after adjustments were made for potential covariates (4). A third casecontrol study did not show any significant association of ROS1 polymorphisms with MI (5).

The studies mentioned above, along with ours, represent another example of failure to replicate previous reports of polymorphisms associated with disease. This may be attributed either to false positive initial associations, or to genuine population diversity. It is possible that for some diseases, the genetic effects of some variants are important in some populations, but not in others (12).

Our study is limited by the small sample size. Thus, we cannot exclude the possibility of a modest effect of rs529038 in disease, which might be apparent in a larger sample size. However, our sample size is similar to that used by Shiffman et al., who initially found the ROS1 polymorphism to be a MI risk factor (3). A power analysis showed that the power of our study to detect the unadjusted OR of 1.75 and the adjusted OR of 1.54 in the Shiffman study is $98 \%$ and $91 \%$, respectively (at a significance level of 0.05 and assuming the additive model of inheritance).

We cannot exclude the possibility of misclassification of subjects with silent CAD in the control group. Some of these individuals were not subjected to coronary angiography or stress testing, and reported the absence of symptoms of disease. Also, cases with fatal MI were not included. Thus, we cannot rule out the possibility that this polymorphism may pre-dispose individuals to more severe disease.

In conclusion, our study did not replicate the findings of Shiffman et al. (3). In the Greek population studied, ROS1 rs529038 polymorphism does not seem to play a major role in the etiology of CAD.
Studies using a larger sample size are needed in order to come up with more definitive conclusions regarding the role of ROS1 rs529038 polymorphism in CAD.

\section{Acknowledgements}

The study was financially supported by: the research project (PENED 03ED474) which was co-financed by E.U.-European Social Fund (75\%) and the Greek Ministry of DevelopmentGSRT $(25 \%)$, by Targeted Financing from the Estonian Ministry of Education and Research (SF0180142s08), by EU FP 7 grant ECOGENE (\#205419, EBC) and by EU grant via the European Regional Development Fund to the Centre of Excellence in Genomics, Estonian Biocentre and University of Tartu.

\section{References}

1. Birchmeier C, Sharma S, Wigler M. Expression and rearrangement of the ROS1 gene in human glioblastoma cells. Proc Natl Acad Sci USA 1987;84:9270-4.

2. Zong CS, Zeng L, Jiang Y, Sadowski HB, Wang LH. Stat3 plays an important role in oncogenic Ros- and insulinlike growth factor I receptor-induced anchorage-independent growth. J Biol Chem 1998;273:28065-72.

3. Shiffman D, Ellis SG, Rowland CM, Malloy MJ, Luke MM, lakoubova $\mathrm{OA}$, et al. Identification of four gene variants associated with myocardial infarction. Am J Hum Genet 2005;77:596-605.

4. Zee RY, Michaud SE, Hegener HH, Diehl KA, Ridker PM. A prospective replication study of five gene variants previously associated with risk of myocardial infarction. $J$ Thromb Haemost 2006;4:2093-5.

5. Horne BD, Carlquist JF, Muhlestein JB, Nicholas ZP, Anderson JL. Associations with myocardial infarction of six polymorphisms selected from a three-stage genomewide association study. Am Heart J 2007;154:969-75.

6. Oguri M, Kato K, Hibino T, Yokoi K, Segawa T, Matsuo $\mathrm{H}$, et al. Genetic risk for restenosis after coronary stenting. Atherosclerosis 2007;194:194-8.

7. Yamada Y, Kato K, Oguri M, Yoshida T, Yokoi K, Watanabe $\mathrm{S}$, et al. Association of genetic variants with atherothrombotic cerebral infarction in Japanese individuals with metabolic syndrome. Int J Mol Med 2008;21:801-8.

8. Yamada Y, Kato K, Yoshida T, Yokoi K, Matsuo H, Watanabe $S$, et al. Association of polymorphisms of ABCA1 and ROS1 with hypertension in Japanese individuals. Int J Mol Med 2008;21:83-9.

9. Braunwald E. Shattuck lecture - cardiovascular medicine at the turn of the millennium: triumphs, concerns, and opportunities. N Engl J Med 1997;337:1360-9.

10. Miller SA, Dykes DD, Polesky HF. A simple salting out procedure for extracting DNA from human nucleated cells. Nucleic Acids Res 1988;16:1215.

11. Krjutskov K, Andreson R, Mägi R, Nikopensius T, Khrunin $A$, Mihailov $E$, et al. Development of a single tube 640-plex genotyping method for detection of nucleic acid variations on microarrays. Nucleic Acids Res 2008;36: e75.

12. loannidis JP, Ntzani EE, Trikalinos TA, ContopoulosIoannidis DG. Replication validity of genetic association studies. Nat Genet 2000;20:306-9. 